\title{
Routing the Messages to Grid Channel Based Model in Wireless Mesh Networks
}

\author{
${ }^{1}$ Ranjit Singh*, ${ }^{2}$ Rakesh Poonia \\ ${ }^{1} \mathrm{Ph} . \mathrm{D}$ Scholar, Tantia University, Sri Ganganagar \\ ${ }^{2}$ Govt. Engineering College, Bikaner \\ Email Id: *ranjitgill345@gmail.com
}

\begin{abstract}
Earlier the work has analyzed and implemented through IPV4 based traffic. The researchers have address the source IPv4 based routing in MRMC method. The router configures with a standard IPV4 based network extracts the source IPV4 address from the packet Header. This paper has been defined the network topology which was work on IPV6 protocol and it may also support IPV4 protocol. This paper implements the equalization that takes observation of channel state. The stations collect channel state information to their neighboring node, later on it was transfer to Gateway. The collected information was only possible through equalization method. In general, the equalization divided into two ways, per symbol and Sequence based which are according to receiver Theory. We are using sequence based theory under equalization that take data from maximum likelihood neighbors. We were used proposed Hybrid that take the existing method and combine with dynamic channel method. The proposed model avoids the multipath propagation problem and that problem only arise when we changes the channel from one wave length to another wavelength. This paper showed proposed results that would be analysis the position of the packets in cluster head $(\mathrm{CH})$. The router is taking as the cluster head which is being deployed on the number of nodes and the se nodes randomly moves from one location to another. The MATLAB Simulator has been used in this research paper that helps to solve the complex mathematical equation. Network Simulator (NS2) has used to implement the Network Model.
\end{abstract}

Keywords - WMNs, Grid, OSPF, CH, BS, Route

\section{INTRODUCTION}

Channel assignment in wireless networks has become an active area of research in these days. The significant issue confronted with WMN is the rare data transfer capacity. For two 802.11 based interfaces to correspond with one another, they have to be allocated to a typical channel. Wireless mesh network [1]system is a guaranteeing remote engineering for a few rising and economically captivating applications. Not quite the same as the conventional system, WMN is dynamic selfarranged, self-composed and self-recuperating. This aspects makes it more appealing with lower in advance expense, less demanding system support work, more powerful systemconstruction modeling and more steady business scope. Basically, an answer for a channel task issue figures out which one of all accessible channels ought to be allocated to a given 802.11 interface.

\section{A. Grid deployment}

There are three categories of grid deployment depending on the layout namely, a rectangular, an equilateral triangle and hexagonal as shown in Fig. 1. Grid based node deployment is an efficient approach for moderate to large scale coverage oriented deployment due to its scalability and simplicity. Deployment of sensor nodes to provide entire area coverage is an important design problem in many wireless Mesh Networks (WMNs) applications. Grid based node deplovment is an efficient and effective practice for provisioning wireless sens or networks. Previous work has reflects grid based deployment of sensor nodes in order to insure sensing coverage under the assumption that each node can be placed exactly at the vertices of the grid.

Among them, we have considered a hexagonal grid because of its normal placement strategy. The nodes are deployed in the hexagonal grid based topologies which are at a fixed distance apart from each other.

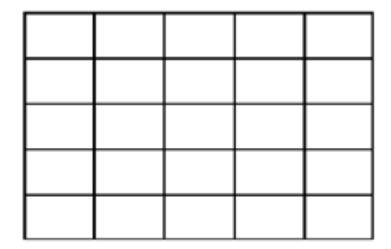

Square Tiles

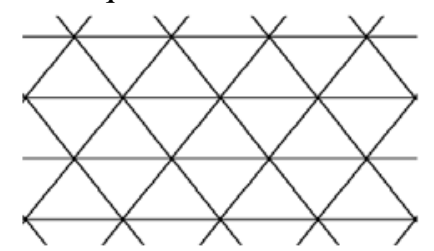

Triangular Tiles

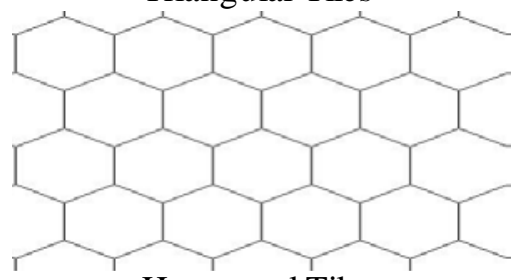

Hexagonal Tiles

Fig..1. Types of Grid Deployment

\section{LITERATURE SURVEY}

J. Wang, W. Shi, K. Cui, F. Jin, and Y. Li (2015) Wireless mesh networks are based on multi-hop standard that enlarge the wireless networks. This technology has many benefits like high bandwidth, fast deployment, easy installation and low maintenance. Despite of these advantages, these wireless networks suffer from interference related problem and which is again solved through allocation of multi-channel to multiple wireless radios. The channel used in multi-radio-multi-channel (MRMC) can be orthogonal channel (OCs), OCs also called as partially overlapped channels (POCs). The advantage of these channels has allocated to appropriate interface so that traffic can move on bidirectional way [2]. 
By default, a wireless device does not have configured MRMC; therefore, by default the router is routed solely based on information within routing table. When an MRMC is applied on the interface, the access point perform additional task of evaluating all network information as they pass through the interface to determine if the information can be forwarded. When network traffic passes through an interface, the access point compares that information in sequential manner, to determine the right packet and this could be done from Cyclic Redundancy Check (CRC).

K. Temma and F. Adachi (2014) availability of channels in wireless networks is limited therefore it affects the overallnetwork due to the less availability of the channels. The solution of this approach would be channel reuse; channel reuse is the method to allocate the same channel to different wireless terminal for transmission perspective. In addition, the availability of channels in wireless networks is limited therefore it affects the overallnetwork due to the less availability of the channels. The solution of this approach would be channel reuse; channel reuse is the method to allocate the same channel to different wireless terminal for transmission perspective [3].

L. Farzinvash and M. Dehghan(2014) Multi-Gateway Multicastrouting (MGMR) [4] is the new approach that fulfill the new requirements of the wireless mesh networks (WMNs). It support multimedia applications that is used for video conferencing and online gaming. These requirements are the current needs of any industry and foremost used in slowest links. The approach will be applying in multiple receivers and adjusted with multiple sessions to track the slowest links.

\section{PROBLEM IDENTIFICATION}

The major issue in $\mathrm{WMN}$ is static channel assignment problem which work on predictable traffic pattern which overload he traffic in between edges and paths. So to overcome the problem, we are focusing in channel assignment problem that reducethe effect of collision and interference and further achieve efficient utilization of channel. Hybrid channel assignment is technique [5]which uses the concept of both static and dynamic allocation schemes.

The proposed methodology is to maintain consistency and uniformity in channel allocation polices and addressing provision of channels and sites for mobile users through comprehensive and unambiguous channel allocation polices. The user who avail data services are the biggest entity of this network system and, unfortunately, the weakest. Consumers pay for the data and subscription fees to avail these data services and are vulnerable against the improper channel allocation[6].

The proposed work has been set up to constantly monitor all the users in a transparent way and ensure a communication level in the network. Large Network with the issue of packet drops, which is basically an abrupt termination of a successful effort to establish a channel. In a mobile node, a path is established successfully only on the allocation of a traffic channel after the required exchange of protocols. The maintenance of this successfully established channel is a vital parameter of Quality of Service[7].

\section{Proposed Model AND EXPERIMENT SETUP}

In this Section, We are identifying the existing static Channel problem, which is also explained in Section-3. The Hybrid Channel is also the solution that we take in this research paper. We are taking the inputs from different nodes and assign dynamic channel to the nodes. The channel has been working on different cases that are explored in more detail:

Case 1: To find the interference; start with first channel $C_{1}$ is sorted way. If (n-1) channels associated with the current network then,

$$
\mathrm{C}_{\mathrm{n}-1} \leq \mathrm{C}_{\mathrm{n}}
$$

If the interference is find out in ' $n$ ' value that means drop ratio of packet is more than the existing channel.

Case 2: To solve collision problem on the network, we
are allocating the random channel by allocating
\[ \mathrm{C}=\mathrm{C}_{\mathrm{n}} / \mathrm{C}_{\mathrm{m}} \]

So this equation takes less collision on the same channel provided to multi-hop networks.

Now, we were taking the Proposed Topology in the figure 2, that behave in above said cases. The topology dynamically configured and condition of channel state depends upon the routing protocol. In our case, the open shortest path first (OSPF) protocol[8] that store the information in server. This information has got from different gateways. Wehave deployed the sensor nodes in the Rectangular field. In rectangular field area, 100 nodes are deployed in $100 \mathrm{~m} * 100 \mathrm{~m}$ area.

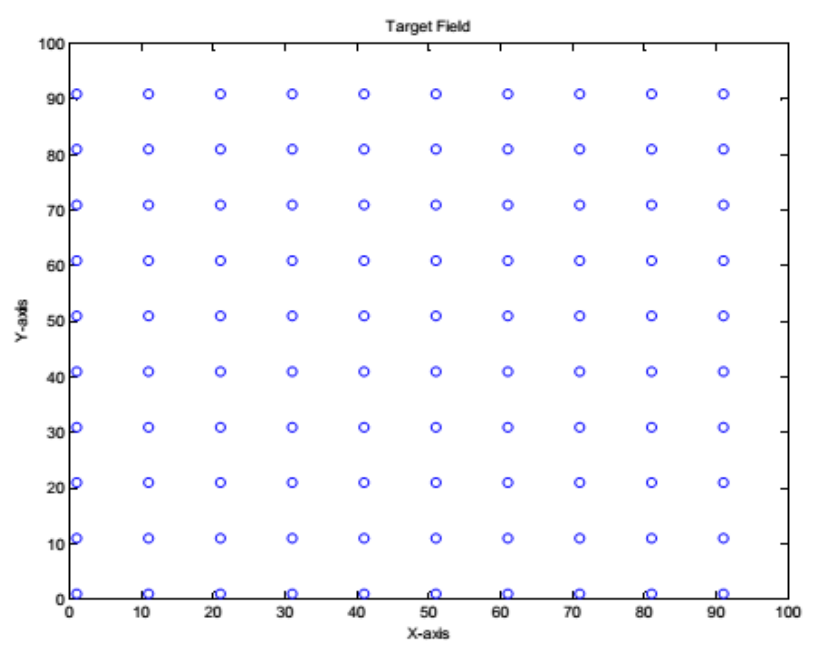

Fig. 2. Uniform Nodes Deployment

The grid based model has represented in figure 3 that share the information to neigbroing nodes. The Cluster Head Selection procedure initialised at that time, if the power of the node was higher, in our case the color of the node was green. This node assumed that it has higher power and work like a Cluster Head. We were using the power management concept from earlier approaches [9]. 


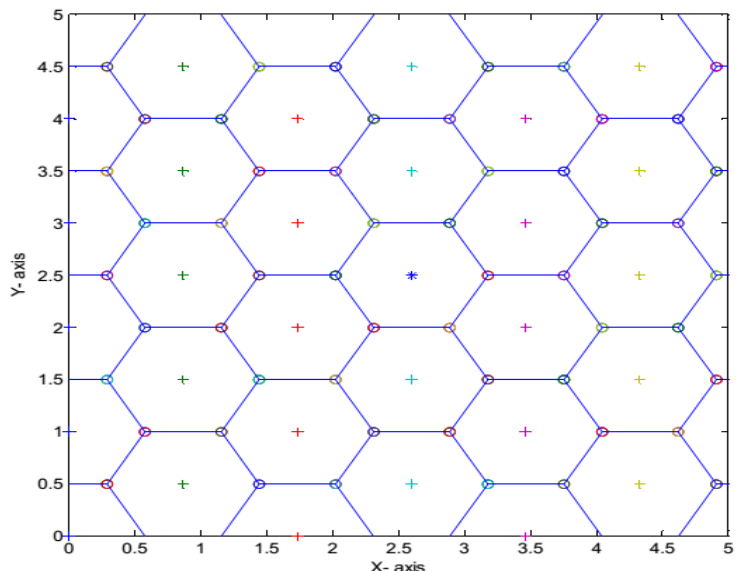

Fig. 3. Hexagonal Grid Topology

The reason for taking the Hexagonal shape from earlier cell spacing approaches [10] and the article clearly mentioned that it can cover larger space. It als o solved the Energy consumption related problems and reduce the number of transmis sions. This way we improve the speed of the network.

\section{RESUlTs AND Discussion}

In the Figure 4, we have investigating the packet, the packet was examined under the network Layer. This packet observed through header structure of IP protocol. Most probably, we have to invest the IPV6 packets. The packet was observed at Base Station (BS) node, this BS behave like the Gateway. The multiple algorithm were running in the device and regularly verify the drop ratio of the packets. In the early study it s end more data because it uses Hexagonal shape in grid based model. Moreover, the speed of the delivery of packet ratio was more than 1000 packet over 1200 rounds. The packet ratio more than 83 percent achieved in this model. We suggest that the proposed model better to work in 100 nodes.

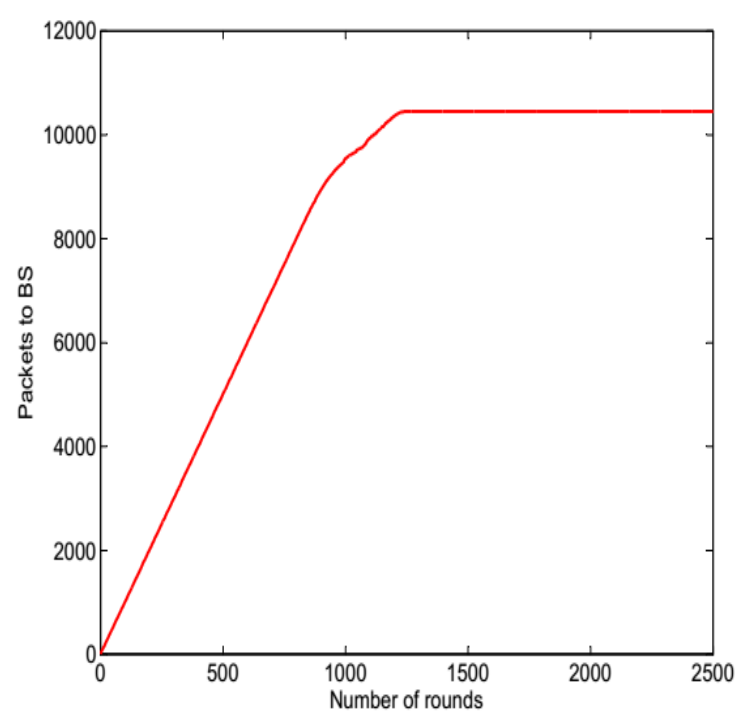

Fig. 4.: Analysis of Packets to BS

In the second case which was represented in figure 5 . The analysis of packets has also studied on cluster based model. The fragmented packets which were 1000 packet. These packets uses multiple directions and move from every cluster, usually average of two or three clusters. The packet ratio that we invested in this case was again more than 83 percent.

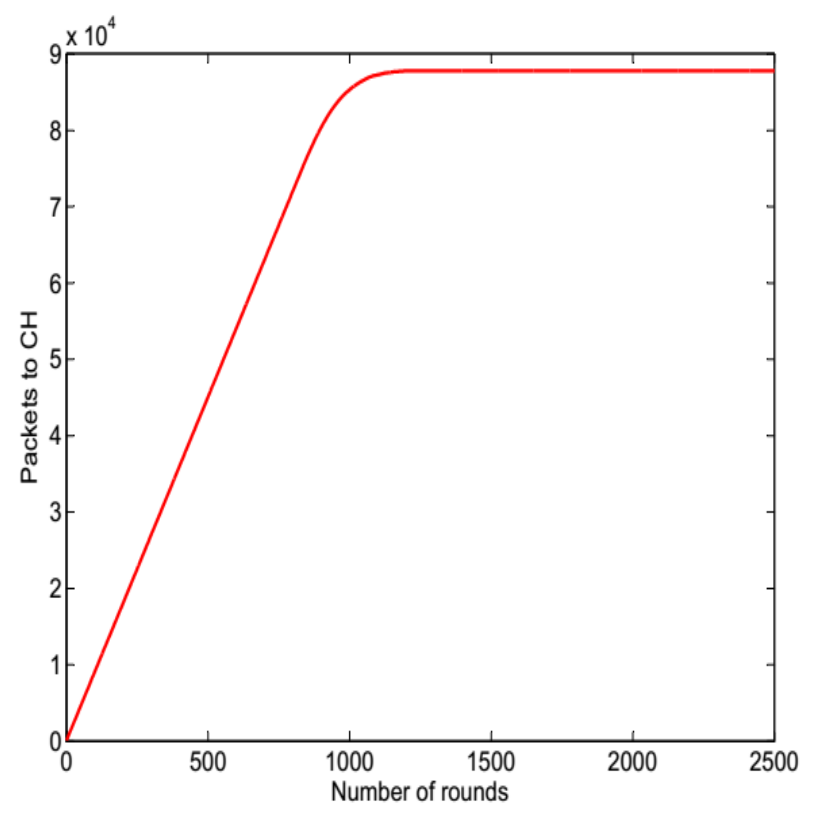

Fig. 5. Analysis of Packets to $\mathrm{CH}$

\section{CONCLUSION AND FUTURE WORK}

This paper observed the packet flow from cluster based network. The observations of the packet really make a lot of study in this work because it gives higher bandwidth. Our aim to deploy better channel in 100 nodes and we were succeed. The investigating results clearly shows that it improves in existing technologies. In future, this work can be extended to increasing the size of network in terms of number of nodes and routers. Moreover, the current work is based on single radio frequency. It can be made to provide topology and collision control for communication at different frequencies within the same network. Moreover, this work is limited to a distributed implementation with no control center. This can be modified in future to accommodate centrally implemented networks. However, there is need to work on power level to remove the interference from the network and to achieve better performance scheme and compare that with this proposed scheme. The work can be extended to determining the traffic overhead and using different techniques to reduce the traffic over channel

\section{REFERENCES}

[1] P. G. Bains, "Comparative Study between Self Reconfigurable and Non Reconfigurable System in WMN," vol. 3, no. 2, pp. 225-229, 2013.

[2] J. Wang, W. Shi, K. Cui, F. Jin, and Y. Li, "Partially Overlapped Channel Assignment for Multi-Channel Wireless Mesh Networks,” EURASIP J. onWireless Commun. Netw., pp. 3770-3775, 2015.

[3] K. Temma and F. Adachi, "Multi-group interference-aware channel segregation based dynamic channel assignment," Proc. 2014 4th IEEE Int. Conf. Netw. Infrastruct. Digit. Content, IEEE IC-NIDC 2014, pp. 51-55, 2014.

[4] L. Farzinvash and M. Dehghan, "Multi-rate multicast routing in multi-gateway multi-channel multi-radio wireless mesh networks," J. Netw. Comput. Appl., vol. 40, no. 1, pp. 
46-60, 2014.

[5] A. U. Chaudhry, R. H. M. Hafez, and J. W. Chinneck, “On the impact of interference models on channel assignment in multi-radio multi-channel wireless mesh networks," Ad Hoc Networks, vol. 27, pp. 68-80, 2015.

[6] C. H. Liu, J. A. Tran, P. Pawelczak, and D. Cabric, "Traffic-aware channel sensing order in dynamic spectrum access networks,” IEEE J. Sel. Areas Commun., vol. 31, no. 11, pp. 2312-2323, 2013.

[7] E. Singla and P. Singh, "Meliorate QOS of WIFIWIMAXin Backhaul Networks," (IJIRCST, vol. 3, no. 1, pp. 89-94, 2015.

[8] E. Xhaferra, "A Review Paper: Analysis of OSPF \& RIPv2 over MPLS VPN with OPNET simulation Edmira Xhaferra,” no. 2, pp. 469-476, 2016.

[9] L. I. U. Kai-ming, M. A. Tao, L. I. U. Yuan-an, and K. O. U. Ke-hao, "Fairness-oriented routing algorithm joint with power control and channel assignment for multi-radio multi-channel wireless mesh networks," J. China Univ. Posts Telecommun., vol. 21, no. 5, pp. 55-60, 2014.

[10] S. Punia, K. Sharma, and C. Kumar, "An Energy Efficient Protocol on Hexagonal Grid-Based Wireless Sensor Network using Super nodes," IJRRA, vol. 1, no. 3, pp. 8085, 2014. 